\title{
BMJ Open Protocol of a longitudinal cohort study on physical activity behaviour in physically disabled patients participating in a rehabilitation counselling programme: ReSpAct
}

\author{
Rolinde A Alingh, ${ }^{1,2}$ Femke Hoekstra, ${ }^{1,2}$ Cees $\mathrm{P}$ van der Schans, ${ }^{2,3}$ \\ Florentina J Hettinga, ${ }^{4}$ Rienk Dekker, ${ }^{2,5}$ Lucas H V van der Woude ${ }^{1,2}$
}

To cite: Alingh RA, Hoekstra F, van der Schans CP, et al. Protocol of a longitudinal cohort study on physical activity behaviour in physically disabled patients participating in a rehabilitation counselling programme: ReSpAct. BMJ Open 2015;5:e007591. doi:10.1136/bmjopen-2015007591

- Prepublication history for this paper is available online. To view these files please visit the journal online (http://dx.doi.org/10.1136/ bmjopen-2015-007591).

Received 6 January 2015 Accepted 13 January 2015

CrossMark

For numbered affiliations see end of article.

Correspondence to

Rolinde A Alingh;

r.a.alingh@umcg.nl

\section{ABSTRACT}

Introduction: Stimulating physical activity behaviour in persons with a physical disability is important, especially after discharge from rehabilitation. A tailored counselling programme covering both the period of the rehabilitation treatment and the first months at home seems on the average effective. However, a considerable variation in response is observed in the sense that some patients show a relevant beneficial response while others show no or only a small response on physical activity behaviour. The Rehabilitation, Sports and Active lifestyle (ReSpAct) study aims to estimate the associations of patient and programme characteristics with patients' physical activity behaviour after their participation in a tailored counselling programme.

Methods and analysis: A questionnaire-based nationwide longitudinal prospective cohort study is conducted. Participants are recruited from 18 rehabilitation centres and hospitals in The Netherlands. 2000 participants with a physical disability or chronic disease will be followed during and after their participation in a tailored counselling programme. Programme outcomes on physical activity behaviour and patient as well as programme characteristics that may be associated with differences in physical activity behaviour after programme completion are being assessed. Data collection takes place at baseline and 14, 33 and 52 weeks after discharge from rehabilitation.

Ethics and dissemination: The study protocol has been approved by the Medical Ethics Committee of the University Medical Centre Groningen and at individual participating institutions. All participants give written informed consent. The study results will provide new insights into factors that may help explain the differences in physical activity behaviour of patients with a physical disability after they have participated in the same physical activity and sports stimulation programme. Thereby, it will support healthcare professionals to tailor their guidance and care to individual patients in order to stimulate physical activity after discharge in a more efficient and effective way. Trial registration number: NTR3961.
Strengths and limitations of this study

- This is the first longitudinal cohort study on a three-phase tailored counselling programme covering the rehabilitation treatment, the transfer from rehabilitation to the home setting and the first months at home.

- The results of this observational study will contribute to the identification of factors that may explain the differences in physical activity behaviour in a large cohort of patients with a variety of physical disabilities and chronic diseases after they have participated in the same physical activity and sports stimulation programme.

- By examining programme characteristics in addition to patient characteristics, more insight can be obtained into the possible association between programme characteristics and patient's physical activity behaviour on short-term and long-term after programme completion.

- Other strengths of this study are that the sample of participants is obtained from 18 different rehabilitation centres and hospitals in The Netherlands that all offer the same programme to their patients, and that participants are evaluated at multiple standardised measurements in time up to 1 year after discharge from rehabilitation.

- Given that patients can only participate in the Rehabilitation, Sports and Active lifestyle (ReSpAct) study if they have been referred to the Sports Counselling Centre, a limitation of the study is a potential sample selection bias by a potential selection bias in the referral.

\section{INTRODUCTION}

Many patients with different types of disabilities and chronic diseases do not obtain the recommended amount of physical activity (PA) that is required for maintaining health. ${ }^{1}{ }^{2}$ In addition, these patients spend considerably more time in sedentary (sitting) 
behaviour compared with the general population, ${ }^{3} 4$ especially those with neuromuscular disabilities and/or those who use a wheelchair for mobility. ${ }^{1}$ Knowing its health-enhancing effects such as the positive effects on mobility and quality of life for persons with a disability, ${ }^{5}$ a physically active lifestyle is strongly recommended for persons with a disability or chronic health condition. ${ }^{3-14}$ Consequently, the promotion of PA behaviour among those persons is of utmost importance. A potentially effective intervention to stimulate long-term participation in PA and sports in persons with a disability or chronic disease is tailored counselling, ${ }^{15-17}$ a form of tailored communication. ${ }^{18}$ Tailored communication is "intended to reach one specific person, based on characteristics that are unique to that person, related to the outcome of interest, and have been derived from an individual assessment". ${ }^{18}$

Last years, several studies ${ }^{19-26}$ have been initiated to investigate the effects of a tailored counselling intervention in different patient populations with the aim of promoting PA behaviour. These studies indicate that the applied postrehabilitation interventions were effective. ${ }^{19}$ 21-23 However, knowing that patients are often faced with many challenges in the transfer from rehabilitation to the home setting and also in engaging in PA and sports (ie, physical, social, attitudinal and financial challenges and barriers) ${ }^{14}{ }^{27-32}$ an intervention that starts during rehabilitation and continues during the transfer to the home setting and the first months at home is perhaps even more effective. ${ }^{33}$ Such a threephase intervention is expected to contribute in maintaining the level of PA in the home setting as acquired during rehabilitation, ${ }^{34}$ and thereby is expected to prevent a postrehabilitation decline in PA and in turn may also counteract a postrehabilitation health decline ${ }^{33}$ Furthermore, when using tailored counselling, such intervention meets the patients' need of professional tailored support at discharge and follow-up, as evidenced by recent studies. ${ }^{31} 32$

As far as known by the authors, only one study ${ }^{17}$ has examined the effects of such a three-phase intervention including tailored counselling and with the aim to stimulate PA behaviour after discharge from rehabilitation. The results from this randomised controlled trial (RCT) indicated that PA behaviour and sport participation were successfully improved up to 1 year after discharge in a heterogeneous inpatient and outpatient population. ${ }^{36}$ However, considerable variation in response was observed in the sense that some patients showed a relevant beneficial response while others showed no or only a small response on PA behaviour. This suggests that the effectiveness of such a programme probably differs between patients, like patients with different ages. ${ }^{17}$ Other factors that are possibly associated with the effectiveness of a tailored counselling programme could be patient's characteristics (eg, diagnosis and stage of behavioural change) and characteristics of the programme (eg, total duration of consultations, extent to which Motivational Interviewing (MI) is applied and treatment form under which the programme is offered). Given the promising results of a three-phase counselling intervention including tailored counselling, ${ }^{36}$ the next step is now to identify factors that may explain the differences in patients' PA behaviour after programme completion. Insight into these factors will contribute to better understand why different patients who all participated in the same programme show different changes in PA behaviour over time. In addition, insight into these factors will help to develop more effective programmes with the aim of stimulating PA behaviour after discharge from rehabilitation, and to offer those programmes in a more efficient way.

A longitudinal cohort study is considered to be an appropriate design to complement the previous RCT study and to perform more in-depth analyses with the aim to identify those explanatory factors. The 'Rehabilitation, Sports and Active lifestyle' study (ReSpAct study; http://www.respact.nl/en) is a nationwide longitudinal cohort study that evaluates the PA and sport stimulation programme 'Rehabilitation, Sports and Exercise' (RSE). This programme is based on the evidence-based programme of van der $\mathrm{Ploeg}^{36}$ and is characterised by both the three-phase intervention set-up and the use of tailored counselling. Since 2012, the RSE programme is being implemented in 18 rehabilitation centres and departments of rehabilitation in general hospitals in The Netherlands and is offered to persons with a variety of physical disabilities and chronic diseases.

The current paper presents the study design of the in-depth scientific evaluation of the RSE programme among patients with a physical disability and/or chronic disease in Dutch rehabilitation care. The main aims of this study at patient level are to gain more insight into patients' PA behaviour and into factors that may help explain differences in patients' PA behaviour after programme completion and at follow-up. For this purpose, both programme outcomes and potential explanatory factors (ie, patient and programme characteristics) are being assessed.

\section{METHODS AND ANALYSIS}

\section{Study design}

The ReSpAct study is a multicentre longitudinal cohort study that is designed to scientifically evaluate the RSE programme. Since 2012, the RSE programme is being implemented and executed within 12 rehabilitation centres and 6 departments of rehabilitation in general hospitals in The Netherlands. Simultaneously, the ReSpAct study is being conducted within these same 18 rehabilitation institutions.

The different subprojects of the ReSpAct study (figure 1) focus on the evaluation of the RSE programme at patient level, the process evaluation of the implementation of the RSE programme within Dutch rehabilitation care $^{37}$ 
Figure 1 Overview of the planned projects within the Rehabilitation, Sports and Active lifestyle (ReSpAct) study. The white boxes indicate the projects that are described in this paper. The grey boxes indicate the projects that are beyond the scope of this paper and are partly presented in Hoekstra et al. ${ }^{37}$ RSE programme=the Rehabilitation, Sports and Exercise programme which is evaluated within the ReSpAct study.

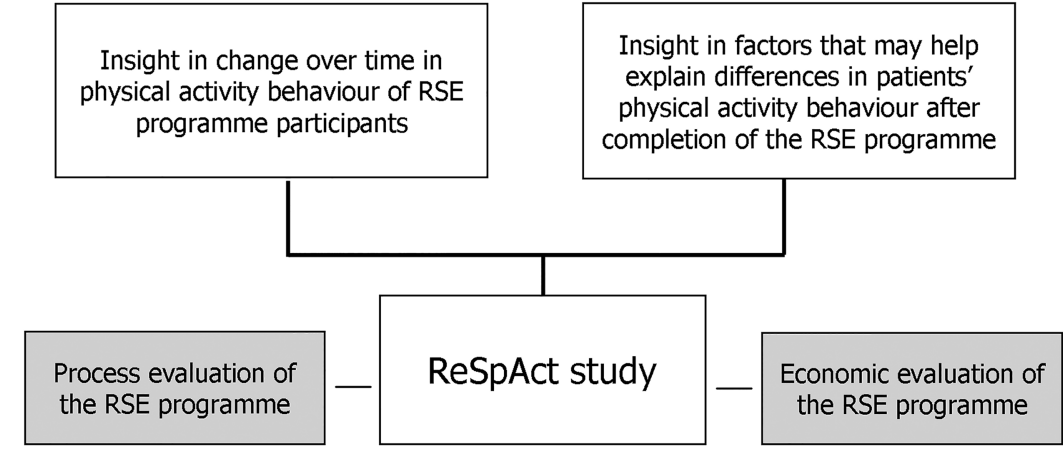

and an economic evaluation of the programme. This paper describes the study protocol of the evaluation of the RSE programme at patient level (figure 1, white boxes).

\section{Study cohort and participating centres}

Participant recruitment began in April 2013 and will continue until October 2015. The ReSpAct study enrols participants with varying physical disability and/or chronic disease who participate in the RSE programme in one of the rehabilitation centres or hospitals involved. Like the general population, the group of participants will be heterogeneous, for example, for age, sex, PA behaviour, as well as for type and extent of disability. Box 1 shows details of eligibility criteria.

\section{Sample size}

PA is the main outcome measure and is assessed using an adapted version of the Short QUestionnaire to ASsess Health enhancing physical activity (SQUASH). ${ }^{38}$ Based on the numbers of patients enrolled in the study of van der Ploeg ${ }^{36}$ it was expected that subgroups of patients of $\mathrm{n}=500$ were deemed necessary and feasible. The ReSpAct study aims to distinguish subgroups of patients based on two-patient characteristics. Based on the context of rehabilitation, a first distinction is made between patients who received rehabilitation care in a rehabilitation centre (12 rehabilitation centres; $n=1000$ )

\section{Box 1 Eligibility criteria}

Inclusion criteria

- Persons, 18 years and older.

- Has a physical disability and/or chronic disease.

- Receives inpatient or outpatient rehabilitation care or treatment based on medicine consultation within one of the participating rehabilitation centres or hospitals.

- Participates in the programme 'Rehabilitation, Sports and Exercise'.

Exclusion criteria

- Not able to complete the questionnaires, even with help.

- Participates in another physical activity stimulation programme. versus patients enrolled from the hospital (6 hospitals; $n=500)$. In addition, subgroups of patients will be formed of inpatients (12 rehabilitation centres; $n=200$ ), outpatients (12 rehabilitation centres and 6 hospitals; $\mathrm{n}=1000$ ) and patients who received treatment based on medicine consultation ( 6 hospitals; $n=300$ ). The target sample size in the ReSpAct study is 1500 patients. To achieve this number, a $25 \%$ attrition rate is factored in the recruitment plans. Therefore the study aims to recruit 2000 patients.

\section{The programme 'RSE'}

Content of the programme

The PA and sport stimulation programme 'RSE' (Dutch: 'Revalidatie, Sport en Bewegen') is a programme aiming to stimulate an active lifestyle in persons with a physical disability and/or chronic disease subsequent to the rehabilitation period. ${ }^{39}$ The stages of change concept of the Transtheoretical model ${ }^{40}$ and the Physical Activity for people with a Disability (PAD) model $^{41}$ formed the theoretical basis for the RSE programme. These models provide insight into the process of behavioural change and the relationships between PA behaviour, its determinants and the daily functioning of persons with a disability.

The RSE programme basically aims to stimulate an active lifestyle during the rehabilitation period and to guide patients in maintaining PA behaviour in the home setting (figure 2). At the start of the rehabilitation treatment, patients' interests and wishes are identified and individual treatment goals are formulated with respect to participation in exercise and sports activities during rehabilitation. Then, by making sports and exercise an integrated part of the individual multidisciplinary treatment (eg, by physiotherapy, adapted PAs and occupational therapy), the patient has the opportunity to become acquainted with various PAs and to get more insight in their own abilities, interests and wishes regarding PAs and sports.

Three to 6 weeks before discharge, the patient is referred to a 'Sports Counselling Centre' (SCG) within a rehabilitation centre or a rehabilitation department of a general hospital. The SCC offers tailored counselling in 


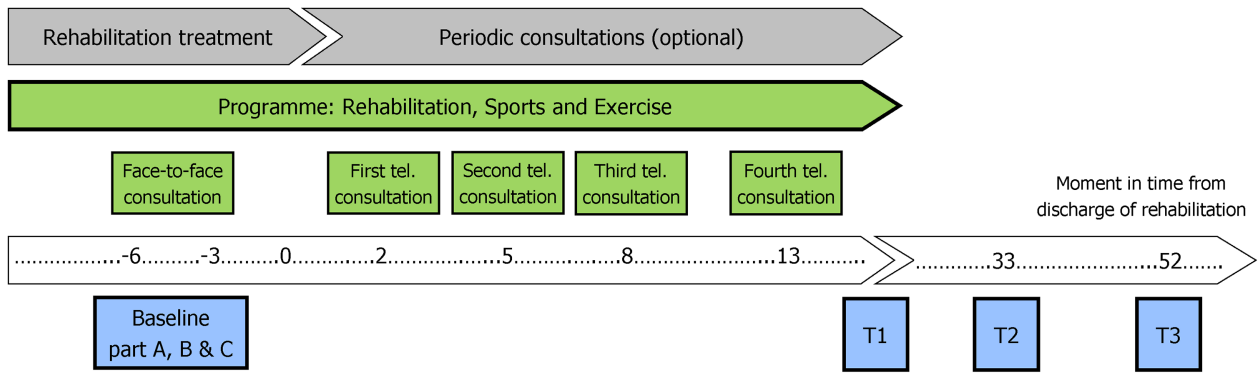

Figure 2 Schematic overview of the components of the programme Rehabilitation, Sports and Exercise (green blocks) and moments of measurement of the Rehabilitation, Sports and Active lifestyle study (blue blocks). The timeline shows in weeks when the various components take place in relation to the moment of discharge of the patient, indicated by 0 .

the form of individual consultations between the patient and a PA and sports counsellor. Counsellors working at the SCC are health professionals specialised in physiotherapy or adapted PA. The consultations at the SCC are intended to guide persons in their behavioural change with the aim to become/be physically active in the home setting after rehabilitation. During patient contact, the trained counsellors make use of MI, which is a client-centred style of conversation. ${ }^{42}$ MI has proven to be effective and seems well suited for use in a variety of healthcare settings. ${ }^{43-51}$

After being referred to the SCC, the patient receives a 30 min individual face-to-face conversation (see figure 2). Dependent on the current patient's stage of behavioural change and patient' motivation for exercise and sports, the topics discussed are tailored to the patient needs. Any personal and environmental barriers and facilitators that may affect the process of behavioural change are also discussed. In addition, the patient receives a booklet on the stages of behavioural change related to an active lifestyle.

Following the first consultation and after discharge from rehabilitation, the patient receives four 10-15 min tailored telephone counselling calls over 13 weeks (see figure 2). With these calls, the patient is further supported in maintaining an active lifestyle in the home environment. Attention is paid to the current PA status and stage of behavioural change. Furthermore, satisfaction with current activities, possible barriers and solutions to barriers are discussed. Offering the telephone counselling calls when the patient is at home again, is in line with the ecological models of PA that suggest that behavioural settings and the broader social context also influence uptake and maintenance of PA behaviour. ${ }^{52}$ Therefore, the home may provide a conducive environment to longer term adherence to PA. ${ }^{15}$ Besides, the patients have to restart and reorganise their lives after discharge, and have to get used to the new situation of having a disability. The counsellor might offer some help in this process of PA behavioural change.

In contrast to the programme of van der Ploeg, ${ }^{36}$ the RSE programme is also appropriate to be implemented in hospitals rather than only within rehabilitation centres. As a result of this, the RSE programme will better suit the complete target group of the rehabilitation care, namely inpatients, outpatients and persons who are treated based on medicine consultations by a rehabilitation physician in the hospital setting. For the latter group of patients, the programme usually consists only of the counselling part, since these patients do not undergo a multidisciplinary rehabilitation treatment.

\section{Counsellors' training in MI}

Within each participating rehabilitation centre and hospital, 1-4 counsellors work at the SCC. All counsellors are referred to follow training in MI by a professional MI trainer, who is a member of the Motivational Interviewing Network of Trainers (MINT). The training consists of a 3-day training course, followed by one refresher-day and biennial refresh-mornings. The 3-day training course provides a comprehensive overview of the foundations of MI, the evidence base concerning behavioural change and the core counselling skills of the approach. The refresher-days aim to involve more deeply in MI. These MI training courses are organised by the Dutch organisation 'Stichting Onbeperkt Sportief' and are offered to all counsellors.

\section{Recruitment}

Patients who have been referred to the SCC and who meet the inclusion criteria regarding age receive written information about the study. During the first consultation at the SCC, the counsellor gives oral information if desired and checks the inclusion and exclusion criteria. Patients who are eligible and willing to participate are asked to sign an informed consent form.

\section{Data collection and outcome measures}

Participants are followed over time through standardised measurements at given regular measurement occasions in time (figure 2). Each measurement consists of filling out a set of questionnaires. The questionnaires can be completed on paper or digitally.

\section{Timeframe of four measurements}

The baseline measurement consists of three parts (table 1). In preparation for the first consultation at the SCC, patients fill out a short questionnaire on their daily 
Table 1 Complete overview on assessments and moments of assessment among participating patients in the ReSpAct study

\begin{tabular}{|c|c|c|c|c|c|c|c|}
\hline Domain & Variables/instruments & $\mathrm{TO}^{*}(\mathrm{~A})$ & $\mathrm{TO}^{*}(\mathrm{~B})$ & $\mathrm{TO}^{*}(\mathrm{C})$ & T1 & T2 & T3 \\
\hline \multicolumn{8}{|c|}{ Primary and secondary outcomes } \\
\hline \multirow[t]{2}{*}{ PA } & Adapted SQUASH, based on refs 3853 & $\mathrm{x}$ & & & $\mathrm{x}$ & $\mathrm{x}$ & $\mathrm{x}$ \\
\hline & $\begin{array}{l}\text { Sport activities as part of rehabilitation care and } \\
\text { self-initiated sport activities }\end{array}$ & $x$ & & & $x$ & $x$ & $x$ \\
\hline Sedentary behaviour & $\begin{array}{l}\text { Transport by motor vehicle, sitting, lying and } \\
\text { sleeping }\end{array}$ & $x$ & & & $x$ & $\mathrm{x}$ & $x$ \\
\hline \multirow[t]{2}{*}{ Health-related quality of life } & Adapted RAND-36, based on refs ${ }^{64-67}$ & & & $x$ & $\mathrm{x}$ & $\mathrm{x}$ & $\mathrm{x}$ \\
\hline & $\begin{array}{l}\text { Visual analogue scale on patient's self-rated } \\
\text { health }\end{array}$ & & & $x$ & $x$ & $x$ & $x$ \\
\hline \multicolumn{8}{|c|}{ Potential factors that help explain differences in patients' PA behaviour-patient characteristics } \\
\hline \multirow[t]{2}{*}{ General } & Demographics and anthropological data & & & $\mathrm{x}$ & & & \\
\hline & Weight and family and living situation & & & $\mathrm{X}$ & $\mathrm{X}$ & $\mathrm{x}$ & $\mathrm{x}$ \\
\hline \multirow[t]{3}{*}{ Lifestyle } & Alcohol use & & & $\mathrm{x}$ & & & $\mathrm{x}$ \\
\hline & Smoking & & & $\mathrm{x}$ & & & $\mathrm{x}$ \\
\hline & Unexpected serious events & & & $x$ & $\mathrm{x}$ & $\mathrm{x}$ & $\mathrm{x}$ \\
\hline \multirow[t]{3}{*}{ Stage of change } & Stage of change (past), based on ref ${ }^{54}$ & & & $x$ & & & \\
\hline & Stage of change (current) ${ }^{54}$ & & $\mathrm{x}$ & & $\mathrm{x}$ & $\mathrm{x}$ & $\mathrm{x}$ \\
\hline & Stage of change & Assesse & ed by $\mathrm{C} \dagger$ & & & & \\
\hline \multirow[t]{8}{*}{ Psychosocial factors } & Self-image related to sports (past) & & & $\mathrm{x}$ & & & \\
\hline & Self-image related to sports (current) & & & & & & $\mathrm{X}$ \\
\hline & Intention & & $\mathrm{X}$ & & $\mathrm{x}$ & $\mathrm{X}$ & $\mathrm{x}$ \\
\hline & Attitude, based on refs 5556 & & $x$ & & $\mathrm{x}$ & $x$ & $x$ \\
\hline & Self-efficacy, based on ref ${ }^{54}$ & & $\mathrm{x}$ & & $\mathrm{x}$ & $\mathrm{x}$ & $\mathrm{x}$ \\
\hline & Perceived social support, based on refs 5758 & & $x$ & & $\mathrm{x}$ & $x$ & $x$ \\
\hline & $\begin{array}{l}\text { Perceived barriers of being physically active, } \\
\text { based on refs } 5960\end{array}$ & & $x$ & & $\mathrm{x}$ & $\mathrm{x}$ & $x$ \\
\hline & Perceived reasons to be physically active & & $\mathrm{x}$ & & $\mathrm{x}$ & $\mathrm{x}$ & $\mathrm{x}$ \\
\hline Motivation & Behavior Exercise Regulation Questionnaire ${ }^{61}$ & & $x$ & & $x$ & $x$ & $x$ \\
\hline \multirow{4}{*}{$\begin{array}{l}\text { Medical status and medical } \\
\text { history }\end{array}$} & Diagnosis & & & $\mathrm{x}$ & & & \\
\hline & Change of medical status & & & & $\mathrm{x}$ & $\mathrm{x}$ & $\mathrm{x}$ \\
\hline & Acceptance of the disease/disability 6869 & & & $\mathrm{x}$ & $\mathrm{x}$ & $\mathrm{x}$ & $\mathrm{x}$ \\
\hline & Physical problems ${ }^{70}$ & & & $\mathrm{x}$ & $x$ & $x$ & $\mathrm{x}$ \\
\hline \multirow[t]{4}{*}{ Fatigue and pacing } & Fatigue Severity Scale 6263 & & $\mathrm{x}$ & & $x$ & $x$ & $x$ \\
\hline & Perceived fatigue & & $\mathrm{x}$ & & & $\mathrm{x}$ & $\mathrm{x}$ \\
\hline & Average activity level & & $\mathrm{x}$ & & & $\mathrm{x}$ & $\mathrm{x}$ \\
\hline & Pacing & & $\mathrm{x}$ & & $\mathrm{x}$ & $\mathrm{x}$ & $\mathrm{x}$ \\
\hline \multicolumn{8}{|c|}{ Potential factors that help explain differences in patients' PA behaviour-programme characteristics } \\
\hline \multirow[t]{3}{*}{ Treatment } & $\begin{array}{l}\text { Context of rehabilitation (rehabilitation centre vs } \\
\text { hospital) }\end{array}$ & Assesse & ed by $\mathrm{C}$ & & & & \\
\hline & Treatment form & Assesse & ed by $\mathrm{C}$ & & & & \\
\hline & Duration of rehabilitation treatment & Assesse & ed by $\mathrm{C}$ & & & & \\
\hline \multirow[t]{2}{*}{ Consultations } & Date, duration and methods of each contact & Assesse & ed by $C$ & & & & \\
\hline & MI applied for each patient contact & Assesse & ed by $\mathrm{C}$ & & & & \\
\hline \multirow{3}{*}{$\begin{array}{l}\text { Patient-counsellor } \\
\text { interpersonal interaction }\end{array}$} & Involvement counsellor in rehabilitation period & & & $x$ & & & \\
\hline & $\begin{array}{l}\text { Patient experience with the face-to-face } \\
\text { consultation }\end{array}$ & & & $x$ & & & \\
\hline & $\begin{array}{l}\text { Patient experience with the telephone } \\
\text { counselling consultations }\end{array}$ & & & & $x$ & & \\
\hline
\end{tabular}

*Baseline measurement consists of three parts: A, B and C.

†C, counsellor.

MI, Motivational Interviewing; PA, physical activity; ReSpAct, Rehabilitation, Sports and Active lifestyle; SQUASH, Short QUestionnaire to ASsess Health enhancing physical activity.

PA (part A). ${ }^{38} 53$ Before the face-to-face conversation starts, patients are asked to fill out another short questionnaire on several psychosocial factors and patient's motivation towards PA behaviour (part B). ${ }^{54-63}$ The last baseline questionnaire is filled out by the patient at home, after the first consultation (part C). ${ }^{54} 64-70$

During the following measurements, the questionnaires are completed as one set (table 1). The second 
measurement (T1) takes place 14 weeks after discharge from rehabilitation to get insight in the direct results of the RSE programme. Patients who receive treatment based on medicine consultation are approached 14 weeks after the first consultation. Follow-up measurements (T2 and T3) are assessed 33 weeks and 1 year after discharge (or after the first consultation).

\section{Content of four measurements}

Each measurement includes a set of validated questionnaires as well as new developed instruments that will be evaluated during data collection. The outcome variables are thoughtfully selected on theoretical and empirical consideration in order to come to a full set of parameters to reach the prescribed aims, while also trying not to challenge participants with too time consuming questionnaires. To check the possibility of completing a measurement within $1 \mathrm{~h}$, and to determine whether all questions were clearly stated, a pilot test was conducted before data collection began. Several persons with a physical disability or chronic disease filled out the baseline questionnaires (ie, part $\mathrm{A}, \mathrm{n}=6$; part $\mathrm{B}, \mathrm{n}=5$; $\operatorname{part} \mathrm{C}, \mathrm{n}=5$ ).

The outcome variables included in the ReSpAct study are summarised in table 1 and are explained in more detail below. In addition, questions about work and medical care consumption are included in order to answer additional research questions (figure 1). Finally, some questions about patients' satisfaction and opinion about the RSE programme are included in the questionnaire as part of the process evaluation of the implementation of the RSE programme. ${ }^{37}$

\section{Primary and secondary outcomes}

Primary outcome: daily PA

Self-reported level of daily PA is the primary outcome of the ReSpAct study and is assessed with an adapted version of the SQUASH. ${ }^{38} 53$ The SQUASH is a selfreported recall questionnaire to assess daily PA of healthy adults based on an average week in the past month. Some minor changes have been made to make the SQUASH applicable for people with a physical disability. Within the domain commuting activities and leisure-time and sports activities, the items 'wheelchair riding' and 'hand biking' have been added. In addition, the self-reported intensity is categorised in 'light', 'moderate' and 'vigorous', instead of 'slow', 'moderate' and 'fast'. Last, 'tennis' has been replaced by '(wheelchair) tennis' at the examples of different sports.

The original SQUASH has proven to be reliable and valid in ordering participants according to their level of PA in an adult population. Acceptable construct validity and moderate-to-high test-retest reliability were found in healthy populations, outpatients with ankylosing spondylitis and patients after a total hip arthroplasty. ${ }^{53} 7172$ Psychometric properties of the adapted version of the SQUASH are investigated in a substudy of the ReSpAct study during data collection.
At baseline, the patients are asked to indicate which PAs they perform in the context of the rehabilitation treatment and on their own initiative.

\section{Secondary outcomes}

Secondary outcomes include daily sedentary behaviour and health-related quality of life (HR-QoL). Sedentary behaviour, such as sitting and lying down, is assessed by a self-reported recall questionnaire which is developed by the authors of the SQUASH ${ }^{53}$ As is carried out in the SQUASH, the activities are assessed based on an average week in the past month. In order to make the questionnaire applicable for wheelchair user patients, these patients are instructed to only count those activities when they are sitting in the wheelchair, rather than actively propelling. HR-QoL is assessed by an adapted version of the RAND-36. ${ }^{646567}$ The RAND-36 is a selfreported questionnaire that assesses eight health concepts from which the physical and mental health summary scores can be derived. Higher scores represent better HR-QoL. ${ }^{64}{ }^{65}$ In addition, a horizontal visual analogue scale was added to record patient's self-rated health. The end points are labelled with 'worst imaginable health status' and 'best imaginable health status'.

\section{Explanatory factors}

To be able to better explain the differences in patients' PA behaviour after completion of the RSE programme, factors that are possibly associated with patients' PA behaviour are examined. Those factors are explained in more detail below on the basis of a subdivision in: patient's characteristics and characteristics of the RSE programme. The selection of the patient's characteristics is based on the PAD model. ${ }^{41}$

\section{Patient's characteristics}

\section{Stages of change}

Provided that the content of the programme is well tailored to the actual stage of behavioural change of the patient, it is expected that the patient's stage of change is a possible predictor of patient's PA behaviour after completion of the programme. To gain more insight into the PA behaviour change, patients are asked to fill out a 'stages of change' questionnaire. Participants have to choose one of five statements, corresponding to the different stages of behavioural change, ${ }^{54}$ according to the Transtheoretical model of Prochaska and DiClemente. ${ }^{40}$ At baseline, this question is answered about both the period before the rehabilitation process was started, and about the current situation. To determine whether the counsellor is able to assess the patient's current stage of change well, the counsellor is asked to register the patient's stage of change too, directly following each consultation.

\section{Psychosocial variables}

Several psychosocial variables are measured over time as possible explanatory variables of PA behaviour. First, the 
behavioural intention to be(come) physically active is assessed with the following item on an 11-point scale: "To what extent do you currently intend to be regularly physically active in the next six months?". Intention is a general concept and an important predictor of behaviour, with planning acting as a mediator. ${ }^{73} 74$

Factors influencing the behavioural intention include attitude, self-efficacy and social influence. ${ }^{75}{ }^{76}$ Attitude towards PA behaviour is assessed with three items, ${ }^{55} 56$ self-efficacy towards PA behaviour with five items out an existing questionnaire (Cronbach's $\alpha=0.82)^{54}$ and two additional items. ${ }^{77}$ To assess perceived social support from family, friends and colleagues concerning PA, seven important items with strong factor loadings were selected from the questionnaire of Sallis $e t a l,{ }^{57}{ }^{78}$ partly based on a previous selection of Papandonatos et al. ${ }^{58}$

The Behavioral Regulation in Exercise Questionnaire (BREQ-2) is used to measure the continuum of behavioural regulation in exercise contexts, ${ }^{61}$ based on the self-determination theory of Deci and Ryan. ${ }^{79}{ }^{80}$ Patients have to respond to 19 items on a five-point Likert scale. The questionnaire showed strong factorial validity in adults who participate in an exercise referral scheme. ${ }^{61}$

In addition, 10 important barriers to and reasons for PA are assessed. The barriers were largely selected from the questionnaire used by van der Ploeg et al. ${ }^{29} 60$

For intention, self-efficacy, attitude, perceived social support and reasons to be physically active a higher score means a more positive value for this variable with respect to a physically active lifestyle. For the barriers to PA a higher score means that the patient experiences the barrier more often.

\section{Fatigue and activity pacing}

Fatigue is a common symptom associated with a wide range of chronic diseases, ${ }^{81} 82$ and one of the strongest predictors of functional disability. ${ }^{83} 84$ In addition, fatigue is seen as a barrier to PA behaviour. ${ }^{27}$ The Fatigue Severity Scale (FSS) is used to assess fatigue. ${ }^{62} 63$ This short questionnaire proves to be a valid and reliable measure to determine the impact of fatigue in several patient samples (eg, multiple sclerosis, cancer, Parkinson's disease, post-stroke and chronic fatigue syndrome $)^{8185}$ and to detect change over time. ${ }^{81}$

As the experienced fatigue also seems to play an important role in determining when and how activities are performed, ${ }^{86}$ the pacing behaviour of performing activities throughout the day ${ }^{87-89}$ is assessed. Since no generic pacing questionnaire is available yet, the different dimensions of the activity of pacing are assessed by a self-constructed nine-item questionnaire that evaluates how and based on what aspects patients modify their PA behaviour over the day.

In addition to the FSS and the pacing questionnaire, perceived fatigue and perceived activity level throughout the day are assessed with a three-item numerical rating scale (range 0-10). The patient is asked to indicate, for an average morning, afternoon and evening of last week, how tired he/she felt and what (daily) activities he/she has performed. Combining the results of these rating scales will give insight into the possible relationship between the activity level and perceived fatigue over the day.

Psychometric properties of both the self-constructed questionnaire on activity pacing and the numerical rating scales will be investigated in a substudy of the ReSpAct study during data collection.

\section{Medical status}

To examine the possible associations between the patient's medical status and patient's PA behaviour after completion of the RSE programme, a number of other variables are assessed. These include: patients' medical status at baseline, changes in medical status over time, patient's physical problems, degree of illness acceptance and unexpected serious events and negative life events. In addition, the self-image of a person's PA is assessed. The instruments used are listed in table 1 .

\section{Characteristics of the RSE programme}

The individual exposure to the RSE programme is dependent on the patient's rehabilitation treatment and the characteristics of the guidance from the SCC. Rehabilitation treatment characteristics (ie, treatment form, duration of the treatment and context of rehabilitation) are registered by the counsellor. Characteristics of the counsellors' guidance includes the methods of contact and the frequency and duration of contact. For each contact, the counsellor registers those aspects of programme dose.

As most counsellors are health professionals specialised in physiotherapy or adapted PA, they often work as a therapist next to their work at the SCC. The possible involvement of the counsellor as a therapist at the rehabilitation treatment of the patient is assessed by one question to the patient. It is expected that involvement of the counsellor as a therapist may positively affect the therapeutic alliance ${ }^{90-92}$ and therefore maybe is positively associated with the patients' PA behaviour after programme completion. ${ }^{93}$

In addition, during each patient contact, the counsellor assesses the degree of MI applied, using a rating scale $(1-10 ; 1=$ really bad, $10=$ very good). It is expected that the extent to which the counsellor applied MI in a consistent way, is positively associated with the behavioural change of the patient. Furthermore, patient experience with the MI-based consultations is assessed using a questionnaire whose psychometric properties will be investigated during data collection.

\section{Planned statistical analyses}

Descriptive characteristics from the participants and the RSE programme will be explored and the univariate correlation structure for the continuous variables will be described. Multilevel analyses comparing subgroups of patients will be conducted on PA behaviour measurements. Multilevel analysis ${ }^{94}$ will be used to analyse repeated measures data because patient data can be 
clustered within the context of rehabilitation (rehabilitation centre vs hospital) and the type of treatment (inpatient vs outpatient rehabilitation care). Furthermore, this technique allows for missing values and can correct for differences at the level of context of rehabilitation and type of treatment, the so-called group effects. If there are any differences at baseline between subgroups of patients, a correction in the analyses will be used for the value of the particular outcome variable at $\mathrm{T}=0$. In addition, possible confounding and interaction effects will be identified and corrected for in the analysis. For all multilevel analyses, the MLwiN (Institute of Education, London, UK) statistical computer programme will be used. Level of significance will be set at $p<0.05$.

\section{DISSEMINATION}

Throughout the ReSpAct study, newsletters will be produced and forwarded to participants and health professionals working at the involved rehabilitation centres and hospitals in order to promote the study and provide updates on its progress. In addition, there is a website launched (http://www.respact.nl) to inform all interested parties about the ReSpAct study.

The results of the ReSpAct study will be disseminated to the scientific, medical and general public. Results will be published in peer-reviewed international journals and will be presented at national and international conferences and symposiums. In addition, results and their practical implications will be disseminated by meetings with clinicians and those interested in rehabilitation care and the stimulation of an active lifestyle among patients with a physical disability or chronic disease.

\footnotetext{
Author affiliations

${ }^{1}$ Center for Human Movement Sciences, University Medical Center Groningen, University of Groningen, Groningen, The Netherlands

${ }^{2}$ Department of Rehabilitation Medicine, Center for Rehabilitation, University Medical Center Groningen, University of Groningen, Groningen, The Netherlands

${ }^{3}$ Research and Innovation Group in Health Care and Nursing, Hanze University of Applied Sciences, Groningen, The Netherlands

${ }^{4}$ Centre of Sport and Exercise Science, School of Biological Sciences, University of Essex, Colchester, UK

${ }^{5}$ Center for Sports Medicine, University Medical Center Groningen, University of Groningen, Groningen, The Netherlands
}

Acknowledgements This study is supported by 'Stichting Onbeperkt Sportief', an organisation that aims for a larger participation of disabled people in sports and physical activity and the development of a suitable and accessible sports facilities.

Contributors All authors contributed to the conception and design of the study. LHVvdW, CPvdS, RD and FJH contributed to obtaining funding. RAA drafted the manuscript. FH, FJH, RD, LHVvdW and CPvdS reviewed the manuscript and provided comments and revisions. All authors read and approved the final manuscript.

Funding This article presents independent research funded by the Dutch Ministry of Health, Welfare and Sports, grant number 319758.

Competing interests None.

Patient consent Obtained.
Ethics approval The Medical Ethical Committee of the University Medical Center Groningen has exempted the approval of the study protocol (reference: M13.131790). Therefore, the ethics committee of the Center of Human Movement Sciences of the University Medical Center Groningen approved the study protocol (reference: ECB/2013.02.28_1). The study was also approved at individual rehabilitation institutions.

Provenance and peer review Not commissioned; internally peer reviewed.

Open Access This is an Open Access article distributed in accordance with the Creative Commons Attribution Non Commercial (CC BY-NC 4.0) license, which permits others to distribute, remix, adapt, build upon this work noncommercially, and license their derivative works on different terms, provided the original work is properly cited and the use is non-commercial. See: http:// creativecommons.org/licenses/by-nc/4.0/

\section{REFERENCES}

1. van den Berg-Emons RJ, Bussmann JB, Stam HJ. Accelerometry-based activity spectrum in persons with chronic physical conditions. Arch Phys Med Rehabil 2010;91:1856-61.

2. Garber C, Blissmer B, Deschenes M, et al. American College of Sports Medicine position stand. Quantity and quality of exercise for developing and maintaining cardiorespiratory, musculoskeletal, and neuromotor fitness in apparently healthy adults: guidance for prescribing exercise. Med Sci Sports Exerc 2011;43:1334-59.

3. Rosenberg DE, Bombardier $\mathrm{CH}$, Hoffman JM, et al. Physical activity among persons aging with mobility disabilities: shaping a research agenda. J Aging Res 2011;2011:708510.

4. Manns PJ, Dunstan DW, Owen N, et al. Addressing the nonexercise part of the activity continuum: a more realistic and achievable approach to activity programming for adults with mobility disability? Phys Ther 2012;92:614-25.

5. Haskell WL, Lee IM, Pate RR, et al. Physical activity and public health: updated recommendation for adults from the American College of Sports Medicine and the American Heart Association. Med Sci Sports Exerc 2007;39:1423-34.

6. Martin Ginis KA, Jorgensen S, Stapleton J. Exercise and sport for persons with spinal cord injury. $P M R$ 2012;4:894-900.

7. Brazzelli M, Saunders DH, Greig CA, et al. Physical fitness training for patients with stroke: updated review. Stroke 2012;43:e39-40.

8. Motl RW, McAuley E. Physical activity, disability, and quality of life in older adults. Phys Med Rehabil Clin N Am 2010;21:299-308.

9. Galea MP. Spinal cord injury and physical activity: preservation of the body. Spinal Cord 2012;50:344-51.

10. Nelson ME, Rejeski WJ, Blair SN, et al. Physical activity and public health in older adults: recommendation from the American College of Sports Medicine and the American Heart Association. Med Sci Sports Exerc 2007;39:1435-45.

11. Durstine JL, Painter P, Franklin BA, et al. Physical activity for the chronically ill and disabled. Sports Med 2000;30:207-19.

12. Verbunt JA, Seelen HA, Vlaeyen JW, et al. Disuse and deconditioning in chronic low back pain: concepts and hypotheses on contributing mechanisms. Eur J Pain 2003;7:9-21.

13. Okawa $Y$, Nakamura $S$, Kudo $M$, et al. An evidence-based construction of the models of decline of functioning. Part 1: two major models of decline of functioning. Int $J$ Rehabil Res 2009;32:189-92.

14. Rimmer JH, Marques AC. Physical activity for people with disabilities. Lancet 2012;380:193-5.

15. Morris $\mathrm{JH}$, Macgillivray S, Macfarlane S. Interventions to promote long-term participation in physical activity after stroke: a systematic review of the literature. Arch Phys Med Rehabil 2014;95:956-67.

16. Leidy NK, Kimel M, Ajagbe L, et al. Designing trials of behavioral interventions to increase physical activity in patients with COPD: insights from the chronic disease literature. Respir Med 2014;108:472-81.

17. van der Ploeg HP, Streppel KR, van der Beek AJ, et al. Successfully improving physical activity behavior after rehabilitation. Am J Health Promot 2007;21:153-9.

18. Kreuter M. What is tailoring? In: Parvanta C, Nelson DE, Parvanta SA, et al, eds. Essentials of public health communication. 1st edn. Jones \& Bartlett Learning, 2010:199-200.

19. Pinto BM, Papandonatos GD, Goldstein MG. A randomized trial to promote physical activity among breast cancer patients. Health Psychol 2013;32:616-26.

20. Ashmore J, Russo R, Peoples J, et al. Chronic obstructive pulmonary disease self-management activation research trial 
(COPD-SMART): design and methods. Contemp Clin Trials 2013;35:77-86.

21. Ang DC, Kaleth AS, Bigatti S, et al. Research to encourage exercise for fibromyalgia (REEF): use of motivational interviewing, outcomes from a randomized-controlled trial. Clin J Pain 2013;29:296-304.

22. Plotnikoff RC, Karunamuni N, Courneya KS, et al. The Alberta Diabetes and Physical Activity Trial (ADAPT): a randomized trial evaluating theory-based interventions to increase physical activity in adults with type 2 diabetes. Ann Behav Med 2013;45:45-56.

23. Jensen LD, Maribo T, Schiottz-Christensen B, et al. Counselling low-back-pain patients in secondary healthcare: a randomised trial addressing experienced workplace barriers and physical activity. Occup Environ Med 2012;69:21-8.

24. Sipila S, Salpakoski A, Edgren J, et al. Promoting mobility after hip fracture (ProMo): study protocol and selected baseline results of a year-long randomized controlled trial among community-dwelling older people. BMC Musculoskelet Disord 2011;12:277.

25. Bennell KL, Egerton T, Bills C, et al. Addition of telephone coaching to a physiotherapist-delivered physical activity program in people with knee osteoarthritis: a randomised controlled trial protocol. BMC Musculoskelet Disord 2012;13:246.

26. Kooijmans H, Post MW, van der Woude LH, et al. Randomized controlled trial of a self-management intervention in persons with spinal cord injury: design of the HABITS (Healthy Active Behavioural Intervention in SCl) study. Disabil Rehabil 2013;35:1111-18.

27. Jaarsma EA, Dijkstra PU, Geertzen $\mathrm{JH}$, et al. Barriers to and facilitators of sports participation for people with physical disabilities: a systematic review. Scand J Med Sci Sports 2014;24:871-81.

28. Kayes NM, McPherson KM, Schluter P, et al. Exploring the facilitators and barriers to engagement in physical activity for people with multiple sclerosis. Disabil Rehabil 2011;33:1043-53.

29. van der Ploeg HP, Streppel KR, van der Beek AJ, et al. Underlying mechanisms of improving physical activity behavior after rehabilitation. Int J Behav Med 2008;15:101-8.

30. Rimmer JH, Riley B, Wang E, et al. Physical activity participation among persons with disabilities: barriers and facilitators. Am J Prev Med 2004:26:419-25.

31. Vissers M, van den Berg-Emons R, Sluis $\mathrm{T}$, et al. Barriers to and facilitators of everyday physical activity in persons with a spinal cord injury after discharge from the rehabilitation centre. $J$ Rehabil Med 2008;40:461-7.

32. Nicholson SL, Donaghy $\mathrm{M}$, Johnston $\mathrm{M}$, et al. A qualitative theory guided analysis of stroke survivors' perceived barriers and facilitators to physical activity. Disabil Rehabil 2013;36:1857-68.

33. Rimmer JH. Getting beyond the plateau: bridging the gap between rehabilitation and community-based exercise. PM R 2012;4:857-61.

34. van den Berg-Emons RJ, Bussmann JB, Haisma JA, et al. A prospective study on physical activity levels after spinal cord injury during inpatient rehabilitation and the year after discharge. Arch Phys Med Rehabil 2008;89:2094-101.

35. Geidl W, Semrau J, Pfeifer K. Health behaviour change theories: contributions to an ICF-based behavioural exercise therapy for individuals with chronic diseases. Disabil Rehabil 2014;36:2091-100.

36. van der Ploeg HP. Promoting physical activity in the rehabilitation setting $[\mathrm{PhD}$ thesis]. VU University Amsterdam, Faculty of Medicine, 2005.

37. Hoekstra F, Alingh RA, van der Schans CP, et al. Design of a process evaluation of the implementation of a physical activity and sport stimulation program in Dutch rehabilitation setting: ReSpAct. Implement Sci 2014;9:127.

38. Wendel-Vos GC, Schuit J. SQUASH: Short QUestionnaire to ASses Health enhancing physical activity. Centrum voor Preventie en Zorgonderzoek, RIVM, 2004

39. Schijvens L, Alingh RA, Duijf M, et al. Handboek Revalidatie, Sport en Bewegen. 2nd edn. Bunnik: Stichting Onbeperkt Sportief, 2014.

40. Prochaska JO, DiClemente CC. Stages and processes of self-change of smoking: toward an integrative model of change. J Consult Clin Psychol 1983;51:390-5.

41. van der Ploeg HP, van der Beek AJ, van der Woude LH, et al. Physical activity for people with a disability: a conceptual model. Sports Med 2004;34:639-49.

42. Miller WR, Rollnick S. Motivational Interviewing: helping people change. 3rd edn. New York: The Guilford Press, 2013.

43. Dunn C, Deroo L, Rivara FP. The use of brief interventions adapted from motivational interviewing across behavioral domains: a systematic review. Addiction 2001;96:1725-42.

44. Burke BL, Arkowitz $\mathrm{H}$, Menchola M. The efficacy of motivational interviewing: a meta-analysis of controlled clinical trials. $J$ Consult Clin Psychol 2003;71:843-61.

45. Hettema J, Steele J, Miller WR. Motivational interviewing. Annu Rev Clin Psychol 2005;1:91-111.
46. Rubak S, Sandbaek A, Lauritzen T, et al. Motivational Interviewing: a systematic review and meta-analysis. Br J Gen Pract 2005;55:305-12.

47. Martins RK, McNeil DW. Review of Motivational Interviewing in promoting health behaviors. Clin Psychol Rev 2009;29:283-93.

48. Armstrong MJ, Mottershead TA, Ronksley PE, et al. Motivational Interviewing to improve weight loss in overweight and/or obese patients: a systematic review and meta-analysis of randomized controlled trials. Obes Rev 2011;12:709-23.

49. Heckman CJ, Egleston BL, Hofmann MT. Efficacy of motivational interviewing for smoking cessation: a systematic review and meta-analysis. Tob Control 2010;19:410-16.

50. Chilton R, Pires-Yfantouda R, Wylie M. A systematic review of motivational interviewing within musculoskeletal health. Psychol Health Med 2012:17:392-407.

51. O'Halloran PD, Blackstock F, Shields N, et al. Motivational interviewing to increase physical activity in people with chronic health conditions: a systematic review and meta-analysis. Clin Rehabil 2014;28:1159-71.

52. Spence JC, Lee RE. Toward a comprehensive model of physical activity. Psychol Sport Exerc 2003;4:7-24.

53. Wendel-Vos GC, Schuit AJ, Saris WH, et al. Reproducibility and relative validity of the short questionnaire to assess health-enhancing physical activity. J Clin Epidemiol 2003;56: 1163-9.

54. Marcus BH, Selby VC, Niaura RS, et al. Self-efficacy and the stages of exercise behavior change. Res Q Exerc Sport 1992;63:60-6.

55. Biddle S, Goudas M, Page A. Social-psychological predictors of self-reported actual and intended physical activity in a university workforce sample. Br J Sports Med 1994;28:160-3.

56. Martilla J, Nupponen R. Assessing attitude: the case of health-enhancing physical activity. Scand J Public Heath 2006;34:168-74.

57. Sallis JF, Grossman RM, Pinski RP, et al. The development of scales to measure social support for diet and exercise behaviors. Prev Med 1987;16:825-36.

58. Papandonatos GD, Williams DM, Jennings EG, et al. Mediators of physical activity behavior change: findings from a 12-month randomized controlled trial. Health Psychol 2012;31:512-20.

59. Sallis JF, Calfas KJ, Alcaraz JE, et al. Potential mediators of change in a physical activity promotion course for university students: project GRAD. Ann Behav Med 1999;21:149-58.

60. Sallis JF, Hovell MF, Hofstetter CR, et al. A multivariate study of determinants of vigorous exercise in a community sample. Prev Med 1989:18:20-34.

61. Markland D, Tobin V. A modification to the behavioural regulation in exercise questionnaire to include an assessment of amotivation. J Sport Exerc Psychol 2004;26:191-6.

62. Krupp LB, LaRocca NG, Muir-Nash J, et al. The fatigue severity scale. Application to patients with multiple sclerosis and systemic lupus erythematosus. Arch Neurol 1989;46:1121-3.

63. Fleurke AM, Goossen WTF, Hoijtink EJ, et al. Fatigue Severity Scale (FSS). http://www.fysiotherapiewetenschap.com/bestanden/31.pdf (accessed Sep 2012)

64. Hays RD, Morales LS. The RAND-36 measure of health-related quality of life. Ann Med 2001;33:350-7.

65. Hays RD, Sherbourne CD, Mazel RM. The RAND 36-Item Health Survey 1.0. Health Econ 1993;2:217-27.

66. van der Zee KI, Sanderman R. Het meten van de algemene gezondheidstoestand met de RAND-36: een handleiding. University of Groningen, University Medical Center Groningen, Research Institute SHARE, 2012.

67. van der Zee KI, Sanderman R, Heyink JW, et al. Psychometric qualities of the RAND 36-item Health Survey 1.0: a multidimensional measure of general health status. Int J Behav Med 1996;3:104-22.

68. Evers AW, Kraaimaat FW, van Lankveld W, et al. Beyond unfavorable thinking: the illness cognition questionnaire for chronic diseases. J Consult Clin Psychol 2001;69:1026-36.

69. Lauwerier E, Crombez G, van Damme S, et al. The construct validity of the illness cognition questionnaire: the robustness of the three-factor structure across patients with chronic pain and chronic fatigue. Int J Behav Med 2010;17:90-6.

70. Beurskens AJ, de Vet HC, Koke AJ, et al. A patient-specific approach for measuring functional status in low back pain. J Manipulative Physiol Ther 1999;22:144-8.

71. Arends S, Hofman M, Kamsma YP, et al. Daily physical activity in ankylosing spondylitis: validity and reliability of the IPAQ and SQUASH and the relation with clinical assessments. Arthritis Res Ther 2013;15:R99.

72. Wagenmakers R, van den Akker-Scheek I, Groothoff JW, et al. Reliability and validity of the short questionnaire to assess 
health-enhancing physical activity (SQUASH) in patients after total hip arthroplasty. BMC Musculoskelet Disord 2008;9:141.

73. Schwarzer R, Lippke S, Luszczynska A. Mechanisms of health behavior change in persons with chronic illness or disability: the Health Action Process Approach (HAPA). Rehabil Psychol 2011;56:161-70.

74. Aizen I. From intentions to actions: a theory of planned behavior. In: Kuhl J, Beckmann J, eds. Action-control: from cognition to behavior. 1st edn. Heidelberg: Springer, 1985:11-39.

75. de Vries H, Dijkstra M, Kuhlman P. Self-efficacy: the third factor besides attitude and subjective norm as a predictor of behavioural intentions. Health Educ Res 1988;3:273-82.

76. de Vries $\mathrm{H}$, Mudde AN. Predicting stage transitions for smoking cessation applying the attitude-social influence-efficacy model. Psychol Health 1998;13:369-85.

77. de Bruin M, Sheeran P, Kok G, et al. Self-regulatory processes mediate the intention-behavior relation for adherence and exercise behaviors. Health Psychol 2012;31:695-703.

78. Stevens M, Bakker-van Dijk A, de Greef MH, et al. A Dutch version of the social support for exercise behaviors scale. Percept Mot Skills 2000;90:771-4.

79. Ryan RM, Deci EL. Intrinsic and extrinsic motivations: classic definitions and new directions. Contemp Educ Psychol 2000;25:54-67.

80. Deci EL, Ryan RM. Intrinsic motivation and self-determination in human behavior. New York: Plenum, 1985

81. Whitehead $\mathrm{L}$. The measurement of fatigue in chronic illness: a systematic review of unidimensional and multidimensional fatigue measures. J Pain Symptom Manage 2009;37:107-28.

82. Swain MG. Fatigue in chronic disease. Clin Sci (Lond) 2000;99:1-8.

83. de Groot MH, Phillips SJ, Eskes GA. Fatigue associated with stroke and other neurologic conditions: implications for stroke rehabilitation. Arch Phys Med Rehabil 2003;84:1714-20.

84. Murphy SL, Lyden AK, Clary M, et al. Activity pacing for osteoarthritis symptom management: study design and methodology of a randomized trial testing a tailored clinical approach using accelerometers for veterans and non-veterans. BMC Musculoskelet Disord 2011;12:177.

85. Elbers RG, Rietberg MB, van Wegen EE, et al. Self-report fatigue questionnaires in multiple sclerosis, Parkinson's disease and stroke: a systematic review of measurement properties. Qual Life Res 2012;21:925-44.

86. Smits BL, Pepping GJ, Hettinga FJ. Pacing and decision making in sport and exercise: the roles of perception and action in the regulation of exercise intensity. Sports Med 2014;44:763-75.

87. White PD, Goldsmith KA, Johnson AL, et al. Comparison of adaptive pacing therapy, cognitive behaviour therapy, graded exercise therapy, and specialist medical care for chronic fatigue syndrome (PACE): a randomised trial. Lancet 2011;377:823-36.

88. Pesek J, Jason L, Taylor R. An empirical investigation of the Envelope Theory. J Hum Behav Soc Environ 2000;3:59-77.

89. Goudsmit EM, Nijs J, Jason LA, et al. Pacing as a strategy to improve energy management in myalgic encephalomyelitis/chronic fatigue syndrome: a consensus document. Disabil Rehabil 2012;34:1140-7.

90. Ackerman SJ, Hilsenroth MJ. A review of therapist characteristics and techniques positively impacting the therapeutic alliance. Clin Psychol Rev 2003;23:1-33.

91. Cheing G, Vong S, Chan F, et al. Testing a path-analytic mediation model of how motivational enhancement physiotherapy improves physical functioning in pain patients. J Occup Rehabil 2014;24:798-805.

92. Vong SK, Cheing GL, Chan F, et al. Motivational enhancement therapy in addition to physical therapy improves motivational factors and treatment outcomes in people with low back pain: a randomized controlled trial. Arch Phys Med Rehabil 2011;92:176-83.

93. Martin DJ, Garske JP, Davis MK. Relation of the therapeutic alliance with outcome and other variables: a meta-analytic review. J Consult Clin Psychol 2000;68:438-50.

94. Snijders TAB, Bosker RJ. Multilevel analysis: an introduction to basic and advanced multilevel modeling. 1st edn. London: Sage Publishers, 1999. 